\title{
Airway bleeding during pulmonary endarterectomy: The "bubbles" technique
}

\author{
Marco Morsolini, MD, ${ }^{\mathrm{a}, \mathrm{b}}$ Andrea Azzaretti, MD, ${ }^{\mathrm{c}}$ Giulio Orlandoni, MD, ${ }^{\mathrm{d}}$ and Andrea M. D'Armini, MD, ${ }^{\mathrm{a}}$ \\ Pavia, Italy
}

Pulmonary endarterectomy (PEA) is a technically demanding operation, currently performed in few centers worldwide. ${ }^{1}$ A bloodless surgical field is mandatory to identify the correct arterial dissection plane, which has to be extended toward subsegmental branches with limited visibility. Vessel perforation is a rare but potentially fatal complication, leading to uncontrollable airway bleeding. A general algorithm for the approach to pulmonary hemorrhage has been proposed by the group from the University of California at San Diego, ${ }^{2}$ whereas alternative managements are only briefly mentioned in the literature. ${ }^{3}$ Extracorporeal life support unloads pulmonary circulation but invariably requires anticoagulation, which is detrimental in pulmonary hemorrhage. We describe a novel technique for the treatment of intraoperative airway bleeding during PEA.

\section{TECHNIQUE}

Generally, PEA can be extended even toward the fifth order of arterial bifurcation. Any arterial disruption at this level cannot be visualized. After arterial dissection is completed, during circulatory arrest, the main pulmonary artery is filled with saline. The patient is ventilated manually while the surgeon checks for the absence of air bubbles coming from the subsegmental arteries. The presence of air bubbles reveals a shunt between airways and the pulmonary circulation. By careful examination it is possible to detect the source of the bubbles and, even if hardly accessible, the injured vessel can be catheterized with a modified syringe for the surgical adhesive delivery. We used a BioGlue Syringe (CryoLife, Inc, Kennesaw, Ga) connected with a graduated $16 \mathrm{Ga} \times 16 \mathrm{~cm}$ single-lumen catheter (Arrow International, Reading, Pa) (Figure 1). Cardiopulmonary bypass is turned on 2 minutes after the adhesive delivery. The absence of air bubbles during manual ventilation indicates the efficacy of the procedure.

\footnotetext{
From Cardiac Surgery, ${ }^{\mathrm{a}}$ Department of Surgery, ${ }^{\mathrm{b}}$ Institute of Radiology, ${ }^{\mathrm{c}}$ and Thoracic Surgery, ${ }^{\mathrm{d}}$ University of Pavia School of Medicine, Foundation "I.R.C.C.S. San Matteo" Hospital, Pavia, Italy.

Disclosures: Authors have nothing to disclose with regard to commercial support.

Received for publication Sept 7, 2012; revisions received Nov 7, 2012; accepted for publication Dec 11, 2012; available ahead of print Jan 11, 2013.

Address for reprints: Marco Morsolini, MD, Division of Cardiac Surgery, University of Pavia School of Medicine, Foundation "I.R.C.C.S. San Matteo" Hospital, Piazzale Golgi, 2-27100, Pavia, Italy (E-mail: m.morsolini@smatteo.pv.it).

J Thorac Cardiovasc Surg 2013;145:1409-10

$0022-5223 / \$ 36.00$

Copyright (c) 2013 by The American Association for Thoracic Surgery

http://dx.doi.org/10.1016/j.jtcvs.2012.12.045
}

Once PEA is completed bilaterally, with the patient's temperature greater than $30^{\circ} \mathrm{C}$, the left ventricular vent is clamped and fiber bronchoscopy is performed to confirm the absence of airway bleeding.

\section{COMMENT}

From April 1994 to October 2012, 434 consecutive patients underwent PEA for chronic thromboembolic pulmonary hypertension. Data were prospectively recorded in a dedicated database approved by the institutional review board. Informed consent was obtained from each patient. The surgical approach for PEA was carried out according to our published technique. ${ }^{4}$

A major prognostic factor for PEA surgery is the experience of the surgeon and the institution. ${ }^{1}$ From 2005 onward, all the operations were performed by the same surgeon. Patient referrals increased substantially over the years, and we became more confident with the procedure. Consequently, our learning curve improved. Overall hospital mortality is $9 \%(40 / 434)$, and postoperative long-term survivals are $86 \%, 80 \%$, and $75 \%$ at 5,10 , and 15 years. The number of episodes of intraoperative airway bleeding requiring immediate intervention is $21(5 \%)$ of 434 , with an intraoperative mortality of $33 \%(7 / 21)$ and a hospital mortality of $48 \%(10 / 21)$.

From April 2009 onward, after we adopted the described "bubble" technique, 2 different mechanisms of bleeding have been recognized, 1 secondary to arterial injury, with a direct arterial-airway shunt, and 1 stemming from systemic collaterals. In case of airway bleeding without air bubbles into the pulmonary artery during manual ventilation, a further clue to check the origin of bleeding is unilateral pulmonary artery crossclamping. If bleeding persists, it necessarily stems from systemic collaterals, which frequently may be found by exploring the pleural cavity. The only treatment for this complication is quick weaning from cardiopulmonary bypass and prompt initiation of procoagulant agents, in addition to differential lung positive pressure. In case of unsuccessful separation from cardiopulmonary bypass, bronchial blocker, embolization, and lung resection may be attempted.

In 4 patients, airway bleeding could be successfully prevented by the surgical adhesive delivery. One of these patients had a fast and uncomplicated recovery, whereas the other 3 had concomitant bleeding owing to multiple systemic collaterals. In these cases, lung resection, pneumonectomy, and bronchial blocker positioning were performed, respectively, with good results except in the patient who, after 


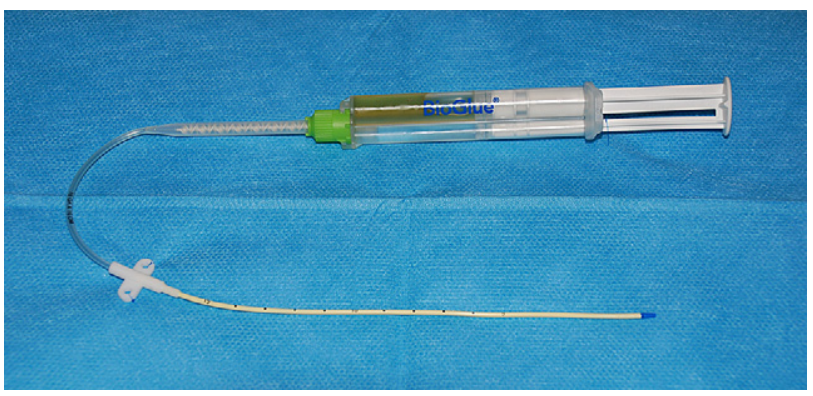

FIGURE 1. Single-lumen catheter.

pneumonectomy, had uncontrollable bleeding from many pleural systemic collaterals.

From 2008 onward, PEA has been applied even in 10 patients for the treatment of pulmonary artery sarcoma. In this peculiar population, air bubbles were noticed more frequently (3/10), probably because the obstructive material is absolutely different in nature and the arterial wall is less strong compared with thromboembolic lesions. Surgical adhesive delivery successfully prevented pulmonary hemorrhage in all patients.
The "bubble" technique has been useful for defining the etiology of airway bleeding intraoperatively and for starting the proper treatment without delay. The identification of an arterial disruption during hypothermic circulatory arrest allows the surgeon to operate immediately. The loss of time caused by the patient's rewarming would be avoided, as well as patient recooling if a surgical repair could be attempted.

\section{References}

1. Mayer E, Jenkins D, Lindner J, D’Armini A, Kloek J, Meyns B, et al. Surgical management and outcome of patients with chronic thromboembolic pulmonary hypertension: results from an international prospective registry. J Thorac Cardiovasc Surg. 2011;141:702-10.

2. Manecke GR Jr, Kotzur A, Atkins G, Fedullo PF, Auger WR, Kapelanski DP, et al. Massive pulmonary hemorrhage after pulmonary thromboendarterectomy. Anesth Analg. 2004;99:672-5.

3. Pretorius V, Alayadhi W, Modry D. Extracorporeal life support for the control of life-threatening pulmonary hemorrage. Ann Thorac Surg. 2009;88: 649-50.

4. Morsolini M, Nicolardi S, Milanesi E, Sarchi E, Mattiucci G, Klersy C, et al. Evolving surgical techniques for pulmonary endarterectomy according to the changing features of chronic thromboembolic pulmonary hypertension patients during 17-year single-center experience. J Thorac Cardiovasc Surg. 2012;144: $100-7$

\title{
Parenchymal-sparing bronchoplasty: A rare surgical approach
}

\author{
Craig Moores, MD, and Darroch Moores, MD, FACS, New Haven, Conn
}

Sleeve lobectomy is a widely accepted surgical approach. One benefit provided by sleeve lobectomy is the salvage of lung parenchyma. This increases its usefulness in patients with seriously limited pulmonary reserve following pneumonectomy. A recent study ${ }^{1}$ determined that wedge bronchoplasty may provide the advantage of decreased postoperative strictures, and preserves native bronchial blood supply without adverse oncologic outcomes. Here, we provide a case report of parenchymal-sparing bronchoplasty that we believe offers the advantage of decreased operative morbidity, preservation of pulmonary reserve, and preservation of native blood supply.

From Yale New Haven Hospital, New Haven, Conn.

Disclosures: Authors have nothing to disclose with regard to commercial support.

Received for publication June 19, 2012; revisions received Aug 22, 2012; accepted for publication Dec 11, 2012; available ahead of print Jan 14, 2014.

Address for reprints: Craig Moores, MD, 50 Elihu St, New Haven, CT 06517 (E-mail: moores.craig@gmail.com).

J Thorac Cardiovasc Surg 2013;145:1410-1

$0022-5223 / \$ 36.00$

Copyright (c) 2013 by The American Association for Thoracic Surgery

http://dx.doi.org/10.1016/j.jtcvs.2012.12.052

\section{CLINICAL SUMMARY}

A 19-year-old man presented to our practice following a syncopal episode and reports of chronic cough. A chest radiograph showed right lower lobe atelectasis. A follow-up computed tomography scan of the chest showed a soft tissue density obliterating the bronchus intermedius with associated complete collapse/consolidation of the right middle and right lower lobes. A flexible bronchoscopy with endobronchial biopsy showed complete obstruction of the proximal bronchus intermedius caused by a low-grade mucoepidermoid tumor.

The following day the patient underwent rigid bronchoscopy with partial endoscopic resection of the tumor. The procedure was terminated due to bleeding and the patient underwent a second debulking 5 days later. It was evident that the tumor arose from the medial aspect of the bronchus intermedius, extending from the level of the right upper lobe distally to the middle lobe orifice (Figure 1). Following debulking, the bronchus intermedius as well as the middle and lower lobe orifices were widely patent. The hope was that the lower and middle lobe atelectasis would resolve with time to allow for a subsequent parenchymal-sparing bronchial sleeve resection. Resolution of atelectasis would result 